\title{
Beneficial effects of pre-stroke statins use in cardioembolic stroke patients with atrial fibrillation: a hospital-based retrospective analysis
}

\author{
Dariusz Kotlęga ${ }^{1}$, Monika Gołąb-Janowska ${ }^{1}$, Agnieszka Meller ${ }^{1}$, Anna Bajer-Czajkowska ${ }^{1}$, \\ Agnieszka Zembroń-Łacny², Przemysław Nowacki ${ }^{1}$, Maciej Banach ${ }^{3}$
}

${ }^{1}$ Department of Neurology, Pomeranian Medical University, Szczecin, Poland
2Department of Applied and Clinical Physiology, University of Zielona Gora,
Zielona Gora, Poland
${ }^{3}$ Cardiovascular Research Centre, University of Zielona Gora, Zielona Gora, Poland

Submitted: 27 February 2018

Accepted: 4 August 2018

Arch Med Sci 2019; 15, 2: 385-392

DOI: https://doi.org/10.5114/aoms.2019.82925

Copyright (c) 2019 Termedia \& Banach

\author{
Corresponding author: \\ Dariusz Kotlęga PhD \\ Department of Neurology \\ Pomeranian Medical \\ University \\ 1 Unii Lubelskiej St \\ 71-252 Szczecin, Poland \\ Phone: +48914253251 \\ E-mail: dkotlega@poczta. \\ onet.pl
}

\begin{abstract}
A bstract
Introduction: Statins are widely used in stroke patients. The AHA/ASA guidelines recommend aggressive statin therapy in atherosclerotic stroke patients. Their beneficial effects are due to both their hypolipemic and pleiotropic properties. The aim of this study was to establish potential benefits from statin use in ischemic stroke patients with the diagnosis of atrial fibrillation (AF).

Material and methods: Ischemic stroke patients with AF were enrolled in the study. Group I, the statin group $(n=181)$, consisted of patients who had been treated with statins before stroke. Group II, the non-statin group $(n=153)$, consisted of patients who had not received such treatment in the last year. In-hospital mortality and neurological deficit on admission and at discharge were analyzed using the National Institutes of Health Stroke Scale (NIHSS) score.

Results: Patients from the non-statin group had greater initial and discharge NIHSS scores (10 vs. 11.9 , probability value $p<0.05 ; 7.6$ vs. $9.5, p<0.05$ respectively). The improvement in NIHSS score was greater in the statin group (73.5\% vs. $59.5 \%, p<0.01)$. In-hospital mortality was more frequent in the non-statin group $(9.9 \%$ vs. $18.3 \%, p<0.05)$.

Conclusions: Despite the predominant use of statins in atherothrombotic stroke patients, we demonstrated the beneficial effects of statins in cardioembolic stroke patients. Detailed cardiovascular screening for statin therapy should be carried out in all AF patients with regard to primary and secondary stroke prevention.
\end{abstract}

Key words: stroke, outcome, atrial fibrillation, anticoagulant prophylaxis, statin, embolism.

\section{Introduction}

Ischemic stroke is a leading cause of death and disability in adults, with the incidence increasing with age. The main cause of stroke is arterial atherothrombosis, which is responsible for $75 \%$ of strokes. Atrial fibrillation (AF) may be present in about $20-25 \%$ of all stroke patients, and in up to $36 \%$ of patients over 80 years of age [1-3]. According to the results of the Framingham Study, the presence of AF increases the incidence of stroke fivefold, which is greater than the increase caused 
by coronary heart disease, hypertension, or cardiac failure [4]. Patients with embolic strokes are at greater risk of death, discharge disability and risk of intracerebral hemorrhage in thrombolytic therapy [5].

The most effective and primary treatment in terms of primary and secondary prevention of cardioembolic stroke is oral anticoagulant therapy [6]. Statins reduce the incidence of stroke (odds ratio, $\mathrm{OR}=0.80 ; 95 \%$ confidence interval (CI): 0.74-0.87; $p<0.00001$ ) [7]. In the primary prevention of stroke, the statin therapy target in individuals without any risk factors is $<160 \mathrm{mg} /$ $\mathrm{dl}$ according to the National Cholesterol Education Program (NCEP) Expert Panel on Detection, Evaluation, and Treatment of High Cholesterol in Adults (Adult Treatment Panel III) recommendations [8]. The American Heart Association/American Stroke Association (AHA/ASA) guidelines for secondary prevention in patients with stroke of 2014 recommend statin therapy with intensive lipid-lowering effects among patients with stroke presumed to be of atherosclerotic origin even when the low-density lipoprotein-cholesterol (LDL-C) level is $<100 \mathrm{mg} / \mathrm{dl}$ with or without any other cardiovascular risk factors [9]. The recommended initiation and goal level of LDL-C is currently decreasing, even to $70 \mathrm{mg} / \mathrm{dl}$ in very high risk patients [10]. According to these guidelines, patients with cardioembolic stroke are not candidates for such treatment. Available data suggest that more factors should be taken into consideration for statin therapy initiation and the goal values of LDL-C such as AF, carotid intima-media thickness, stress or chronic kidney disease [11-13].

Over the years, it has become increasingly evident that the lower the LDL-C levels are, the greater are the benefits for stroke patients. In spite of the beneficial effects of aggressive statin therapy in stroke prevention proven by the Stroke Prevention by Aggressive Reduction in Cholesterol Levels (SPARCL) study, such treatment should not be generalized to include nonatherosclerotic stroke patients because cardioembolic stroke patients were excluded from the study [14-16]. The study even suggested that statin therapy should not be initiated in cardioembolic stroke patients unless other cardiovascular indications are evident [16].

It is worth noting that the main focus has been on the atherosclerotic pathomechanism of stroke; however, there are available data suggesting a potentially important role of statins in embolic stroke patients. Theoretically, the beneficial effect of statins may result from the pleiotropic properties that reflect the interactions within the immune system and inflammatory molecules. This group of drugs is proven to exert multidirectional effects, including effects on reactive oxygen species devel- opment, clot formation, and brain plasticity [17]. The first observations linking the histological findings and the presence of inflammation in AF were presented by Frustaci et al. [18]. The presence of inflammatory markers such as C-reactive protein (CRP) is associated with the presence of AF and predicts the future development of arrhythmias. However, it is not clear whether the initiation of AF activates direct inflammatory effects or whether the presence of a preexisting systemic inflammatory state promotes further persistence of AF [19]. It has been demonstrated that premorbid statin use is connected with higher grades of collateral circulation detected on cerebral angiography [20]. There are studies where the authors assessed the efficacy of statin therapy in AF patients. The potentially beneficial effects of statins in paroxysmal AF patients were not observed in relation to mortality and AF recurrence [21]. In a 5-year follow-up, the non-atherosclerotic stroke patients who had statins prescribed at discharge were at lower risk of death and future cardiovascular events [22]. The beneficial effects of statins have been proven in the primary prevention of stroke. In the largest meta-analysis of pre-stroke statin use, an improved outcome was observed at 30 and 90 days, but there was no AF subgroup analysis [23]. There is limited information about the potential use and effectiveness of pre-stroke statins in embolic stroke patients. Different factors may increase the in-hospital mortality in stroke patients; thus, we also considered this [24].

The aim of the study was to analyze the impact of pre-stroke statin use on in-hospital outcomes and mortality in cardioembolic stroke patients.

\section{Material and methods}

Characteristics of patients. The retrospective analysis included medical documentation of ischemic stroke patients $(n=2000)$ who were screened based on their history of AF. The additional inclusion criterion was the availability of information regarding statin use before stroke. All patients were Caucasians. We included 334 of 565 screened patients with AF-originated cardioembolic stroke after exclusion of other known causes of stroke and cardioembolism. The exclusion criterion was a lack of information regarding prestroke statin use. Group I, the statin group, consisted of 181 patients who had been treated with statins directly before the stroke, while group II, the non-statin group ( $n=153)$ was composed of patients who had not undergone such treatment. The population sample size was sufficient for the analysis with anticipated AF incidence of $25 \%$; confidence level (CL) 95\%; and $\mathrm{Cl} 5 \%$. Death as an endpoint was used in the primary group, for which at least 536 patients were needed. Analysis 
was performed on the basis of information available from patient history, additional test results, and other medical records. The medical history was obtained from medical documentation and self-reports of patients. Participants underwent standard physical examination and laboratory and additional tests. Patients were treated according to the same guidelines of conventional medical practice. The definition of stroke used was that provided by the World Health Organization (WHO) $[25,26]$. The AF was defined as any type of AF detected during hospitalization or confirmed by previous medical records. Statins were used in different doses. Three types of statins were used: atorvastatin, simvastatin, and rosuvastatin.

According to the Local Ethical Committee, approval was not needed (decision number KB0012/226/05/18).

\section{Statistical analysis}

The objective of this trial was to investigate the alternative hypothesis that pre-stroke statin use positively influences the in-hospital outcome and mortality in cardioembolic stroke patients (the biological null hypothesis was that there were no significant improvements in stroke patients who used statins within the pre-hospital period). Two main types of variables were assessed: measurement and nominal variables. To assess the equality of variances of variables (age, total cholesterol, LDL cholesterol, high-density lipoprotein (HDL) cholesterol, triglycerides (TG), NIHSS score), the Brown-Forsyth test was used before a comparison of means. The test did not show significance for age or the National Institutes of Health Stroke Scale (NIHSS) score variables; hence, a two-sample $t$-test was used for age and NIHSS assessment. Because of the skewness and non-normality of the distributions between the remaining nominal variables (Shapiro-Wilk test), the results were presented as a number (percentage) for qualitative variables or a median for quantitative variables. These data, which included data for the two groups, were compared using the nonparametric Mann-Whitney $U$-test and the exact Fisher test. Multivariable logistic regression models including patients' age, sex, arterial hypertension, ischemic heart disease, previous myocardial infarction, diabetes mellitus, smoking, previous stroke or transient ischemic attack, previous anticoagulants usage, initial lipid profile, and international normalized ratio (INR) as independent variables were used to identify independent predictors of lack of improvement, progression, or death.

A value of $p<0.05$ was considered to indicate statistical significance. All statistical analyses were performed with Statistica 12 software.

\section{Results}

The characteristics of the analyzed groups in terms of the main risk factors for stroke (both groups consisted of AF patients) are presented in Table I. Significantly lower LDL-C, total cholesterol and TG plasma concentrations were found in the statin group. There was also a slight difference in the HDL-C levels between the groups, but this was not significant. We did not find any differences between the groups with regard to other cardiovascular risk factors. The statin therapy was continued in all statin group patients and introduced, due to their lipid profiles, in $60.3 \%$ of patients from the non-statin group. At least 2 points were scored on the $\mathrm{CHA}_{2} \mathrm{DS}_{2}$ VASc scale by $93.6 \%$ of patients in the statin group and by $93.9 \%$ of patients in the non-statin group (nonsignificant difference).

We observed a significantly lower neurological deficit, using the NIHSS, on admission and at discharge from hospital in the statin group than in the non-statin group (Table II).

Intrahospital recovery after stroke (NIHSS score on discharge vs. initial NIHSS score) was found to be significantly more frequent in AF patients treated with statins before stroke onset (Figure 1).

In-hospital mortality was $9.9 \%$ in the statin group and $18.3 \%$ in the non-statin group, a significant difference (Figure 2). The causes of death in the groups were as follows: stroke - 10 patients, pulmonary embolism - 1 patient, myocardial infarction - 2 patients, circulatory insufficiency 4 patients, chronic renal failure -1 patient (statin group); and stroke - 14, myocardial infarction - 4, circulatory insufficiency -7 , bilateral pneumonia 1 , chronic renal failure -2 individuals (non-statin group).

A multivariable logistic regression analysis was performed to identify independent predictors of lack of improvement, neurological progression, or death. We found that female sex and coronary heart disease (CHD) were predictors of absence of change or worsening of condition on the NIHSS during hospitalization in the statin group, while age and previous stroke were the corresponding predictors in the non-statin group (Table III). The predictors of death during hospitalization were age, CHD, and diabetes in the statin group and age alone in the non-statin group (Table III).

\section{Discussion}

We presented an analysis of ischemic stroke patients suffering from embolic stroke. Despite the primary interest and research in statin therapy being with regard to the atherosclerotic pathomechanism of stroke, there has been emerging evidence of potential beneficial effects of this group of drugs in cardioembolic stroke patients. 
Table I. Characteristics of groups in terms of the main risk factors for stroke

\begin{tabular}{|c|c|c|c|}
\hline Risk factor & Statin group $(n=181)$ & Non-statin group $(n=153)$ & $P$-value \\
\hline Age, median, range [years] & $80,70-85^{\#}$ & $78,72-84^{\#}$ & $0.384^{*}$ \\
\hline \multicolumn{4}{|l|}{ Gender, $n(\%)$ : } \\
\hline Male & $59(32.6)$ & $39(25.5)$ & $0.155^{*}$ \\
\hline Female & $122(67.4)$ & $114(74.5)$ & \\
\hline Arterial hypertension, $n(\%)$ & $162(89.5)$ & $137(89.5)$ & $0.995^{*}$ \\
\hline Ischemic heart disease, $n(\%)$ & $111(61.3)$ & $90(58.8)$ & $0.694^{*}$ \\
\hline Previous myocardial infarction, $n(\%)$ & $28(15.5)$ & $26(17)$ & $0.811^{*}$ \\
\hline Diabetes mellitus, $n(\%)$ & $64(37.4)$ & $46(31.1)$ & $0.329^{*}$ \\
\hline Smoking, $n(\%)$ & $29(16.1)$ & $26(17.2)$ & $0.863^{*}$ \\
\hline Previous stroke, $n(\%)$ & $41(22.7)$ & $27(17.9)$ & $0.454^{*}$ \\
\hline \multicolumn{4}{|l|}{ Number of strokes, $n(\%)$ : } \\
\hline 1 & $29(16.1)$ & $20(13.2)$ & $0.450^{*}$ \\
\hline 2 & $8(4.4)$ & $5(3.3)$ & $0.527^{\star *}$ \\
\hline 3 & $3(1.7)$ & $4(2.6)$ & $0.435^{\star \star}$ \\
\hline Previous transient ischemic attack, $n(\%)$ & $8(4.4)$ & $4(2.7)$ & $0.782^{\star \star}$ \\
\hline Previous anticoagulants, $n$ (\%) & $12(6.7)$ & $6(4)$ & $0.673^{\star \star}$ \\
\hline Previous antiaggregants, $n$ (\%) & $68(37.6)$ & $65(42.9)$ & $0.886^{*}$ \\
\hline Previous anti-diabetic drugs, $n(\%)$ & $45(24.9)$ & $37(24.2)$ & $0.494^{*}$ \\
\hline Previous antihypertensives, $n(\%)$ & $101(55.8)$ & $127(83)$ & $0.991^{*}$ \\
\hline \multicolumn{4}{|l|}{ Lipids profile (min.-max.; mean \pm SD) [mg/dl]: } \\
\hline Total cholesterol & $84-298 ; 176.5 \pm 40.87$ & $82-318 ; 178 \pm 43.79$ & $0.003^{* * *}$ \\
\hline LDL cholesterol & $30-224 ; 106.6 \pm 34.61$ & $32-252 ; 108 \pm 36.78$ & $<0.001^{* * *}$ \\
\hline HDL cholesterol & $12-118 ; 48.9 \pm 16.40$ & $21-114 ; 47.5 \pm 14.89$ & $0.472^{* * *}$ \\
\hline Triglycerides & $36-284 ; 108.9 \pm 42.05$ & 39-394; $109.7 \pm 46.11$ & $<0.001^{* * *}$ \\
\hline \multicolumn{4}{|l|}{ BMI (min.-max.; mean $\pm \mathrm{SD}$ ) $\left[\mathrm{kg} / \mathrm{m}^{2}\right]$ : } \\
\hline Male & $17-45 ; 27.8 \pm 7.27$ & $18-30.5 ; 23.9 \pm 4.21$ & $0.081^{*}$ \\
\hline Female & $18-42.3 ; 27.4 \pm 5.71$ & $18-45 ; 28.2 \pm 5.83$ & $0.343^{*}$ \\
\hline ICA significant stenosis, $n(\%)$ & $26(14.8)$ & $20(13.8)$ & $0.880^{*}$ \\
\hline Therapeutic INR, $n$ (\%) & $10(5.5)$ & $6(3.9)$ & $0.801^{\star *}$ \\
\hline
\end{tabular}

"Quartiles Q1-Q3; *Mann-Whitney U test; **exact Fisher test; ***Student's t-test.

Table II. Initial and discharge NIHSS score in patients included in groups I and II

\begin{tabular}{|lccc|}
\hline NIHSS & Group I $(n=181)$ & Group II $(n=153)$ & $P$-value \\
\hline $\begin{array}{l}\text { Initial (min.-max.; } \\
\text { mean } \pm \text { SD) }\end{array}$ & $3-38 ; 10.0 \pm 8.58$ & $4-33 ; 11.9 \pm 8.40$ & $0.024^{*}$ \\
\hline $\begin{array}{l}\text { On the day of discharge } \\
\text { (min.-max.; mean } \pm \text { SD) }\end{array}$ & $0-38 ; 7.6 \pm 8.59$ & $0-33 ; 9.5 \pm 8.92$ & $0.035^{*}$ \\
\hline
\end{tabular}

${ }^{*}$ Two-sample t-test. 


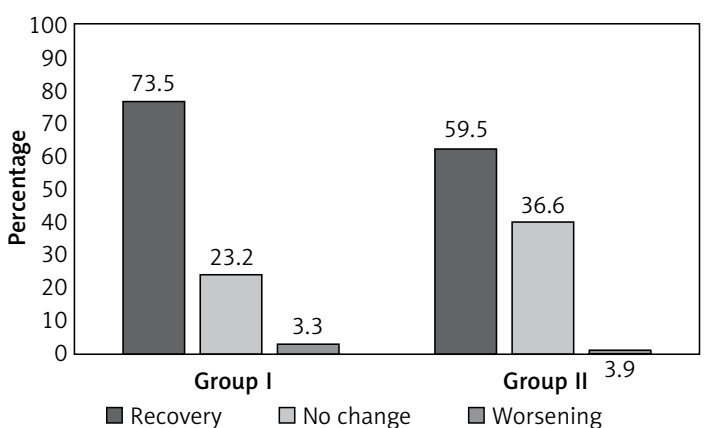

Figure 1. Intrahospital recovery after stroke in AF patients treated with statins before stroke onset. Recovery: group I vs. group II, $p=0.0067$; no change: group I vs. group II, $p=0.0074$; worsening: group I vs. group II, $p=0.7666$

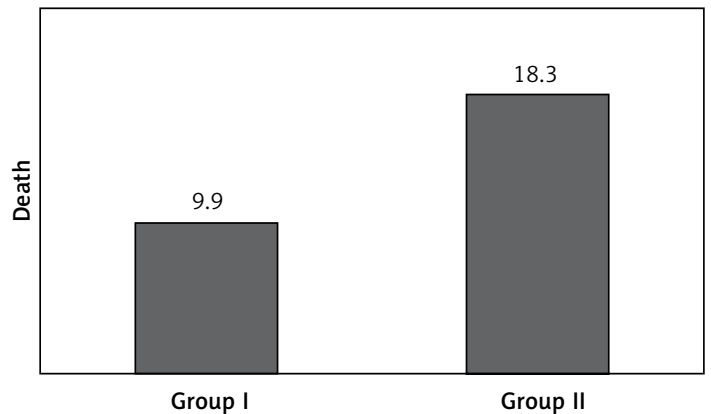

Figure 2. Significantly more frequent death in AF patients not treated with statins before stroke onset (group I vs. group II; $p=0.0203$ )

Table III. Multivariable regression of predictors in groups

\begin{tabular}{|c|c|c|c|c|}
\hline Parameter & $P$-value & $\begin{array}{l}-95 \% \mathrm{CL} \\
+95 \% \mathrm{CL}\end{array}$ & Odds ratio & $\begin{array}{l}-95 \% \mathrm{CL} \\
+95 \% \mathrm{CL}\end{array}$ \\
\hline Female sex* & 0.01 & $\begin{array}{l}0.25 \\
1.91\end{array}$ & 2.94 & $\begin{array}{l}1.29 \\
6.75\end{array}$ \\
\hline $\mathrm{CHD}^{*}$ & 0.02 & $\begin{array}{c}-1.7 \\
-0.19\end{array}$ & 0.38 & $\begin{array}{l}0.17 \\
0.83\end{array}$ \\
\hline $\mathrm{Age}^{\star \star}$ & 0.02 & $\begin{array}{c}0.007 \\
0.09\end{array}$ & 1.05 & $\begin{array}{c}1.0 \\
1.09\end{array}$ \\
\hline Previous stroke** & 0.02 & $\begin{array}{l}0.13 \\
1.93\end{array}$ & 2.81 & $\begin{array}{l}1.14 \\
6.89\end{array}$ \\
\hline $\mathrm{Age}^{\star \star \star}$ & 0.01 & $\begin{array}{l}0.02 \\
0.17\end{array}$ & 1.1 & $\begin{array}{l}1.02 \\
1.19\end{array}$ \\
\hline$C H D^{\star \star \star}$ & 0.03 & $\begin{array}{l}-4.38 \\
-0.21 \\
\end{array}$ & 0.1 & $\begin{array}{l}0.01 \\
0.81\end{array}$ \\
\hline Diabetes $^{\star * *}$ & 0.004 & $\begin{array}{l}-20.9 \\
-0.04\end{array}$ & 0.31 & $\begin{array}{c}0.1 \\
0.96\end{array}$ \\
\hline Age $^{\star \star \star \star *}$ & 0.01 & $\begin{array}{l}0.02 \\
0.14\end{array}$ & 1.08 & $\begin{array}{l}1.02 \\
1.15\end{array}$ \\
\hline
\end{tabular}

*Predictors of a lack of improvement or worsening in NIHSS during hospitalization in statin group; ** predictors of a lack of improvement or worsening in NIHSS during hospitalization in non-statin group; ${ }^{* * *}$ predictors of death during hospitalization in statin group; ${ }^{* * *}$ predictors of death during hospitalization in non-statin group.

In our group of patients, we observed significantly lower plasma concentrations of LDL-C, total cholesterol, and TG in the statin group. A slight difference in the HDL-C levels was also present between the groups, but this result was not significant. We did not observe any differences between the groups with regard to other cardiovascular risk factors. In our opinion, the significant differences were probably due to the hypolipemic effect of statins used in statin group individuals, which was a consequence of the inclusion criterion. We did not analyze the possible confounding effect of the treatment of cardiovascular disorders.

We observed a significantly lower neurological deficit in NIHSS on admission and at discharge from the hospital in the statin group than in the non-statin group (Table II). These results are clinically important, as there were no differences in the classical cardiovascular risk factors besides the lipid profile and history of statin use. The most logical explanation for the observed difference in neurological deficit between the two groups is the effect of statins. The effect of statins on neurological deficit in stroke patients may also come from the pleiotropic activity of statins [27]. This activity was observed in a group of stroke patients with initially low LDL-C levels who were later administered statins and compared to non-users of statins in the pre-stroke period. The first group had better functional outcomes [28].

In our analysis, we found the incidence of $\mathrm{AF}$ to be $28.2 \%$ in stroke patients, which is similar to the reports from other authors in this region [3]. The beneficial effects of statin therapy at the time of stroke onset have been revealed in several studies [23]. To our knowledge, the only study that was focused on AF stroke patients and pre-stroke statin use $(n=1030)$ showed a $32 \%$ reduction in 
the frequency of severe stroke $(\mathrm{OR}=0.68 ; 95 \% \mathrm{Cl}$ : $0.5-0.92 ; p=0.011)$ defined as a modified Rankin scale (mRs) score $\geq 4$ points at 30 days [29].

In our analysis, we also concentrated on the in-hospital mortality. In the statin group it was $9.9 \%$, while in the non-statin group it was as high as $18.3 \%$; the difference was statistically significant. Taken together with the results presented above, we also observed the beneficial effects of statin use in the pre-stroke period with regard to mortality. There was an analysis performed of stroke patients in the follow-up lasting 22.2 months [30]. The mortality rate in this period was lower in the statin users than in those not using statins. The effect was observed in both low- and high-potency statin groups, but therapy did not affect the stroke recurrence rate [31]. On the other hand, mortality was not related to statin use in paroxysmal AF patients without stroke [21]. The lack of such effects in non-stroke patients may confirm the importance of the pleiotropic effects of statins in stroke patients as there are multidirectional, inflammatory cascades activated on stroke onset. In the follow-up of patients who had suffered stroke and were subsequently treated with statins compared to non-users, there was no difference between the groups in the risk of recurrent stroke. The only beneficial effect of statins was the reduced risk of death during any hospitalization in the follow-up period [32].

We also analyzed the predictors of lack of improvement or neurological decline during hospitalization. The predictors with the greatest impact were a history of previous stroke in the non-statin group and female sex in the statin group (OR 2.81 and 2.94 respectively). The analysis of negative predictors of death showed that age had the greatest impact in both groups of patients. In the statin group CHD and diabetes were additionally detected as negative predictors of death, which is an interesting observation when considering that this group usually had better outcomes. We conclude that taking statins is one of the independent factors negatively affecting prognosis in stroke patients.

We have shown that the administration of statins before stroke is associated with a lower frequency of circulatory insufficiency as a cause of death, which can be linked to the cardioprotective effect of statins. This effect may be responsible for the low mortality of the statin group.

The effect of statins was not affected by oral anticoagulant therapy, as we did not observe differences between the groups in relation to anticoagulant therapy or frequency of achieved therapeutic INR. There was a slightly higher frequency of anticoagulant treatment before hospitalization in the statin group, but this difference was not significant. If this correlation had been confirmed on a greater scale and with statistical significance, then anticoagulant therapy would have been considered as a factor affecting outcome in the statin group.

We also found a very small proportion of patients who had used oral anticoagulants despite the presence of indications according to the $\mathrm{CHA}_{2} \mathrm{DS}_{2}$ VASc scale. In the years 2010 to 2013, this scale was not widely used in Poland and the knowledge of such a need was not sufficient. According to our findings, we suggested detailed screening for cardiovascular risk factors beside the $\mathrm{CHA}_{2} \mathrm{DS}_{2}$ VASc scale and initiated statin therapy in all suitable patients with AF in regard of primary stroke prevention [33]. The prevalence of dyslipidaemia in Poland is $60-70 \%$ in adults [34]. It seems to be of importance as patients with $\mathrm{AF}$ may be undermanaged in terms of statin prescription. This may apply to all AF patients and patients with a history of embolic stroke as a means of secondary stroke prevention [35]. Ischemic stroke patients with AF should be stratified more carefully, especially given that epidemiological trends indicate a decrease in the atherosclerotic risk factor profile associated with an increasing tendency for cardiac embolism [36].

The safety of potential intensified statin therapy in primary stroke prevention of AF patients needs to be established, although it may be entirely safe for intravenously thrombolyzed patients [37].

The limitation of our study is the lack of differentiation between the duration, doses, and types of statins that were used by the patients. The differences in statin pharmacokinetics and strength of dosage may be important factors affecting the results. The limitation of the mortality analysis may be the size of the group, because in calculating the dichotomous endpoint, there should be at least 536 patients (268 in each group). We surpassed that number in the primary analysis with 565 patients, but the final number of included patients was less than optimal. However, it is too early to draw a clear conclusion on a clinically relevant relationship between pre-stroke statin use and in-hospital mortality in stroke patients due to the small sample size. The sample size may have also played a role in the appearance of some confounding factors, and differences between the groups could have been observed had the analysis been performed on a larger group. A difference between the groups regarding statin use during hospitalization may have also affected our results.

We cannot ignore that the observed results may only be reflective of superior general health care status of statin group patients, although we did not reveal any other significant differences between the groups. We also did not perform a post-hospital follow-up of mortality and mRs for neurological deficit assessment. 
In conclusion, the use of statins in the prestroke period seems to be beneficial with regard to risk of death and neurological deficit in the acute phase of stroke. The final impact on stroke survival and outcome is a consequence of effects of multiple factors, and statins may be one of them. Despite the predominant use of statins in atherothrombotic strokes, we demonstrated the beneficial effects of statins in cardioembolic stroke patients. We suggest detailed cardiovascular screening for statin therapy in all AF patients with regard to primary and secondary stroke prevention.

\section{Conflict of interest}

The authors declare no conflict of interest.

\section{References}

1. Sienkiewicz-Jarosz H, Głuszkiewicz M, Pniewski J, et al. Zapadalność i wskaźnik śmiertelności dla pierwszego w życiu udaru mózgu - porównanie dwóch warszawskich badań populacyjnych. Neurol Neurochir Pol 2011; 45: 207-12.

2. Kotlęga D, Ciećwież S, Turowska-Kowalska J, Nowacki P. Pathogenetic justification of statin use in ischaemic stroke prevention according to inflammatory theory in development of atherosclerosis. Neurol Neurochir Pol 2012; 46:176-83.

3. Gołąb-Janowska M, Meller A, Kotlęga D, Bajer-Czajkowska A, Nowacki P. Atrial fibrillation and stroke - coexistence and attitude to preventive therapy on the basis of Szczecin and Szczecin region patients. Neurol Neurochir Pol 2014; 48: 410-5.

4. Wolf PA, Abbott RD, Kannel WB. Atrial fibrillation as an independent risk factor for stroke: the Framingham Study. Stroke 1991; 22: 983-8.

5. Saposnik G, Gladstone D, Raptis R, Zhou L, Hart RD; the Investigators of the Registry of the Canadian Stroke Network (RCSN) and the Stroke Outcomes Research Canada (SORCan) Working Group. Atrial fibrillation in ischemic stroke: predicting response to thrombolysis and clinical outcomes. Stroke 2013; 44: 99-104.

6. van Walraven C, Hart RG, Singer DE, et al. Oral anticoagulants vs aspirin in nonvalvular atrial fibrillation: an individual patient meta-analysis. JAMA 2002; 288: 2441-8.

7. Wang W, Zhang B. Statins for the prevention of stroke: a meta-analysis of randomized controlled trials. PLoS One 2014; 9: e92388.

8. Executive Summary of The Third Report of The National Cholesterol Education Program (NCEP) Expert Panel on Detection, Evaluation, and Treatment of High Blood Cholesterol in Adults (Adult Treatment Panel III). JAMA 2001; 285: 2486-97.

9. Kernan WN, Ovbiagele B, Black HR, et al.; American Heart Association Stroke Council, Council on Cardiovascular and Stroke Nursing, Council on Clinical Cardiology, and Council on Peripheral Vascular Disease. Guidelines for the Prevention of Stroke in Patients With Stroke and Transient Ischemic Attack. A Guideline for Healthcare Professionals From the American Heart Association/ American Stroke Association. Stroke 2014; 45: 2160-236.

10. Soran H, Adam S, Mohammad JB, et al. Hypercholesterolaemia - practical information for non-specialists. Arch Med Sci 2018; 14: 1-21.
11. Zanchetti A, Liu L, Mancia G, et al. Blood pressure and LDL-cholesterol targets for prevention of recurrent strokes and cognitive decline in the hypertensive patient: design of the European Society of Hypertension-Chinese Hypertension League Stroke in Hypertension Optimal Treatment randomized trial. J Hypertens 2014; 32: 1888-97.

12. Banach M, Mikhailidis DP, Kjeldsen SE, Rysz J. Time for new indications for statins? Med Sci Monit 2009; 15: MS1-5.

13. Cheng HG, Patel BS, Martin SS, et al. Effect of comprehensive cardiovascular disease risk management on longitudinal changes in carotid artery intima-media thickness in a community-based prevention clinic. Arch Med Sci 2016; 12: 728-35.

14. Collins R, Armitage J, Parish S, Sleight P, Peto R. Effects of cholesterol-lowering with simvastatin on stroke and other major vascular events in 20536 people with cerebrovascular disease or other high-risk conditions. Lancet 2004; 363: 757-67.

15. Amarenco P, Bogousslavsky J, Callahan A III, et al.; Stroke Prevention by Aggressive Reduction in Cholesterol Levels (SPARCL) Investigators. High-dose atorvastatin after stroke or transient ischemic attack. N Engl J Med 2006; 355: 549-59.

16. Furie KL. High-dose statins should only be used in atherosclerotic strokes. Stroke 2012; 43: 1994-5.

17. Kotlęga D, Gołąb-Janowska M, Masztalewicz M, Ciećwież S, Nowacki P. Potential role of statins in the intracerebral hemorrhage and subarachnoid hemorrhage. Neurol Neurochir Pol 2015; 49: 322-8.

18. Frustaci A, Chimenti C, Bellocci F, Morgante E, Russo MA, Maseri A. Histological substrate of atrial biopsies in patients with lone atrial fibrillation. Circulation 1997; 96: 1180-4.

19. Engelmann MD, Svendsen JH. Inflammation in the genesis and perpetuation of atrial fibrillation. Eur Heart J 2005; 26: 2083-92.

20. Lee MJ, Bang OY, Kim SJ, et al. Role of statin in atrial fibrillation-related stroke: an angiographic study for collateral flow. Cerebrovasc Dis 2014; 37: 77-84.

21. Watanabe E, Yamashita T, Suzuki S, et al.; Investigators TJ. Statin treatment for patients with paroxysmal atrial fibrillation. Int Heart J 2011; 52: 103-6.

22. Ntaios G, Papavasileiou V, Makaritsis K, et al. Statin treatment is associated with improved prognosis in patients with AF-related stroke. Int J Cardiol 2014; 177: 129-33.

23. Ní Chróinín D, Asplund K, Åsberg S, et al. Statin therapy and outcome after ischemic stroke: systematic review and meta-analysis of observational studies and randomized trials. Stroke 2013; 44: 448-56.

24. Snarska KK, Bachórzewska-Gajewska H, Kapica-Topczewska K, et al. Hyperglycemia and diabetes have different impacts on outcome of ischemic and hemorrhagic stroke. Arch Med Sci 2017; 13: 100-8.

25. Adams Jr HP, del Zoppo G, Alberts MJ, et al.; American Heart Association/American Stroke Association Stroke Council; American Heart Association/American Stroke Association Clinical Cardiology Council; American Heart Association/American Stroke Association Cardiovascular Radiology and Intervention Council; Atherosclerotic Peripheral Vascular Disease Working Group; Quality of Care Outcomes in Research Interdisciplinary Working Group. Guidelines for the early management of adults with ischemic stroke: a guideline from the American Heart Association/American Stroke Association stroke 
council, clinical cardiology council, cardiovascular radiology and intervention council, and the atherosclerotic peripheral vascular disease and quality of care outcomes in research interdisciplinary working groups: the American Academy of Neurology affirms the value of this guideline as an educational tool for neurologists, Circulation 2007; 115: e478-534.

26. Hatano S. Experience from a multicentre stroke register: a preliminary report. Bull World Health Organ 1976; 54: 541-53.

27. Heart Protection Study Collaborative Group. MRC/BHF Heart Protection Study of cholesterol lowering with simvastatin in 20536 high-risk individuals: a randomised placebo-controlled trial. Lancet 2002; 360: 7-22.

28. Stead LG, Vaidyanathan L, Kumar G, et al. Statins in ischemic stroke: just low-density lipoprotein lowering or more? J Stroke Cerebrovasc Dis 2009; 18: 124-7.

29. Ko D, Thigpen JL, Otis JA, et al. Influence of statin therapy at time of stroke onset on functional outcome among patients with atrial fibrillation. Int J Cardiol 2017; 227 : 808-12.

30. Choi JY, Seo WK, Kang SH, et al. Statins improve survival in patients with cardioembolic stroke. Stroke 2014; 45: 1849-52.

31. Ntaios G, Papavasileiou V, Makaritsis K, et al. Statin treatment is associated with improved prognosis in patients with AF-related stroke. Int J Cardiol 2014; 177: 129-33.

32. Wu YL, Saver JL, Chen PC, et al. Effect of statin use on clinical outcomes in ischemic stroke patients with atrial fibrillation. Medicine (Baltimore) 2017; 96: e5918.

33. Zembala M, Filipiak K, Kowalski O, et al. Staged hybrid ablation for persistent and longstanding persistent atrial fibrillation effectively restores sinus rhythm in longterm observation. Arch Med Sci 2017; 13: 109-17.

34. Banach M, Jankowski P, Jóźwiak J, et al. PoLA/CFPiP/PCS guidelines for the management of dyslipidaemias for family physicians 2016. Arch Med Sci 2017; 13: 1-45.

35. Xia SJ, Du X, Li C, et al. Uptake of evidence-based statin therapy among atrial fibrillation patients in China: a report from the CAFR (Chinese Atrial Fibrillation Registry) Study. Int J Cardiol 2016; 220: 284-9.

36. Bogiatzi C, Hackam DG, McLeod AI, Spence JD. Secular trends in ischemic stroke subtypes and stroke risk factors. Stroke 2014; 45: 3208-13.

37. Tsivgoulis G, Kadlecová P, Kobayashi A, et al. Safety of statin pretreatment in intravenous thrombolysis for acute ischemic stroke. Stroke 2015; 46: 2681-4. 